Journal of Turbulence

Vol. 00, No. 00, 00 00, 1-22

\title{
The signature of laminar instabilities in the zone of transition to turbulence
}

\author{
N. Vinod \& Rama Govindarajan \\ Engineering Mechanics Unit, \\ Jawaharlal Nehru Centre for Advanced Scientific Research, \\ Jakkur, Bangalore 560064 INDIA \\ e-mail: nvinod@jncasr.ac.in, rama@jncasr.ac.in \\ (November 13, 2018)
}

We demonstrate that the space-time statistics of the birth of turbulent spots in boundary layers can be reconstructed qualitatively from the average behavior of macroscopic measures in the transition zzone. The conclusion in [1] that there exists a connection between the patterns in laminar instability Jand the birth of turbulent spots is strengthened. We examine why the relationship between instability - and transition to turbulence is manifest in some cases and appears to be totally absent in others. Novel cellular automaton type simulations of the transition zone are conducted, and the pattern of spot Birth is obtained from secondary instability analysis. The validity of the hypothesis of concentrated breakdown, according to which most turbulent spots originate at a particular streamwise location, is' assessed. The predictions made lend themselves to straightforward experimental verification.

\section{Introduction}

N.

The physics of the transition to turbulence in a boundary layer has been the subject of a large number of theoretical and experimental investigations. While most of the process is understood, as shown in the schematic picture in figPure 11 there are still gaps in our knowledge, as we shall discuss below. The Jprocess begins with the linear amplification of two-dimensional disturbance $\rightarrow$ waves of a narrow band of frequencies. Once the disturbance modes have grown to a certain amplitude, they destabilize three-dimensional secondary -modes [2-4]. These modes display a locally regular pattern of maxima, which - may be aligned (harmonic) or staggered (subharmonic). Further downstream, in what is termed the transition zone, turbulent spots, or concentrated patches Tof turbulence, are found, surrounded by quiet laminar flow. The spots grow as - the convect downstream, and merge with each other to make the flow asymptotically fully turbulent. Our interest here is in the breakdown of instability waves into turbulent spots. We do not explain the complex physics occurring between the zone of secondary instability and the region containing spots, but 


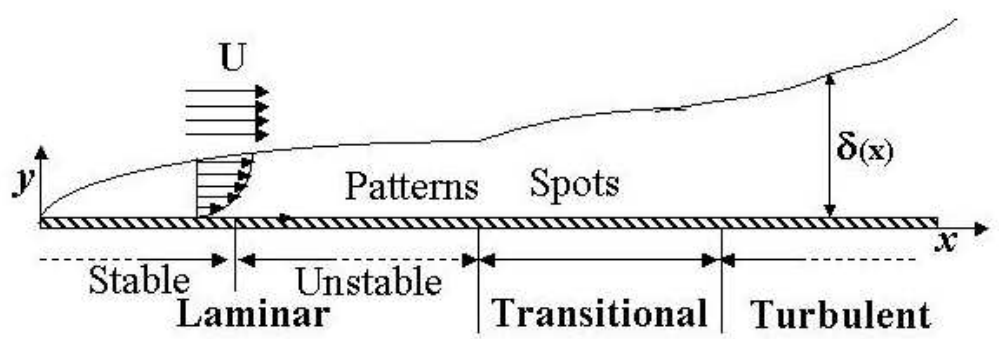

Figure 1. Sequence of events in the laminar-turbulent transition process, on a boundary layer formed by the flow past a semi-infinite plate.

we present evidence showing that the pattern existing in the first is retained for the most part in the second.

It is at present almost impossible either experimentally or numerically to track the birth and downstream growth of a large number of individual turbulent spots and to obtain detailed statistics. It is however not difficult to measure average quantities such as turbulent intermittency (the fraction of time that a flow is turbulent). With these, and knowing the behaviour of a typical spot, we demonstrate that it is possible to reconstruct the scenario of spot birth. It was recently shown [1] that intermittency behaviour in many flows is consistent with spot birth as dictated by the most unstable secondary mode. We present another example of this in decelerating flows. Hitherto, it was assumed that spot birth is completely random. No contradiction to this assumption was presented by intermittency measurements in low external noise environments, in the flow past a flat plate aligned with the flow. Since most intermittency measurements were made for this specific flow, the assumption of random spot birth gained credence and was not re-examined. We show that in this special case, the intermittency variation obtained from both random and regular spot birth are similar, and discuss why. We then present other measures which even in this case would differentiate clearly between random and regular spot birth. We exploit the versatility of our simulation method to demonstrate (section 7) that the effect of geometry (in the form of transverse curvature) is significant in the boundary layers on cylinders. It is hoped that the present predictions will motivate experimental efforts.

We conduct secondary instability computations in the standard manner (section 2), and employ novel cellular automaton type stochastic simulations for spot birth, growth and convection, as described in section 3 . We make a number of assumptions. First, while the secondary instability analysis is threedimensional, the stochastic simulations are conducted in a plane parallel to the solid body, when viewed from above. This means that the variations within the boundary layer in a direction $y$ normal to the wall are averaged over. The 
$x-y$ profile of turbulent spots is not uniform, but given that they are flat objects and the boundary layer thickness is very small $\left(O\left(R^{-1}\right)\right.$ compared to the other dimensions), averaging over $y$ is a valid approximation. Secondly, we employ the hypothesis of concentrated breakdown, according to which all spots are born at a particular streamwise location [5], i.e. at a particular Reynolds number. We show in section [6 that this is a reasonable assumption. We also make use of the experimentally and numerically observed shape and downstream behaviour of single spots, especially the fact that spot growth is self-similar at any pressure gradient.

A spatial pattern in turbulent in the form of spirals and spots are observed in the experiments of Prigent et al. [6] in plane Couette flow and Taylor-Couette flow. They found that the spatial modulation of turbulent intensity obeys the dynamics of coupled amplitude (Ginzburg-Landau type) equations with noise. It is important to note that the simulations of $[7,8]$ in boundary layers establish that structures in the unstable laminar region are the precursors of turbulent spots. These simulations were of bypass transition. While the present work is about the traditional Tollmien-Schlichting mechanism taking place in low-disturbance environments, the basic connection between instability and transition is similar.

\section{Analysis of secondary instability}

The growth of small (linear) perturbations in a boundary layer, under the assumption of parallel flow, is described by the Orr-Sommerfeld equation $[9,10]$

$$
(U-\omega / k)\left(v^{\prime \prime}-k^{2} v\right)-U^{\prime \prime} v=\frac{1}{1 k R}\left(v^{\mathrm{iv}}-2 k^{2} v^{\prime \prime}+k^{4} v\right),
$$

where $U(y)$ is the mean velocity and $R$ the Reynolds number, $\equiv U_{\infty}(x) \theta(x) / \nu$, $U_{\infty}$ is the local freestream velocity, $\theta$ is a characteristic boundary layer thickness, and $\nu$ the kinematic viscosity. $v(y)$ is the amplitude of the normal component $\hat{v}$ of the disturbance velocity, given by

$$
\hat{v}(x, y, t)=v(y) \exp [1(k x-\omega t)]
$$

$k$ and $\omega$ being the wavenumber and the frequency of the disturbance mode being considered. The primes denote differentiation with respect to $y$.

When the linear modes have grown to a significant amplitude (of the order of a percent of the mean flow), the new flow becomes unstable to secondary, typically three-dimensional, disturbances. The linearised equations describing the secondary instability are obtained by writing flow quantities in the form 
$[11,12]$

$$
\mathbf{U}(x, y, t)=\{U(y) \vec{i}+A[u(y) \vec{i}+v(y) \vec{j}] \exp [1(k x-\omega t)]\}+\mathbf{u}_{s}(x, \vec{y}, z, t)
$$

The quantity in the curly brackets is the basic flow consisting of the laminar profile plus the primary instability wave. The primary instability is very slowly growing, and the flow may be taken to be periodic. $A$ stands for the amplitude of the primary disturbance. The three-dimensional secondary disturbance is written as

$\mathbf{u}_{s}\left(y, \mathbf{r}_{\perp}, t\right)=\operatorname{Re}\left\{\mathbf{u}_{s+}(y) \exp \left[1\left(\mathbf{k}_{+} \cdot \mathbf{r}_{\perp}-\omega_{+} t\right)\right]+\mathbf{u}_{s-}(y) \exp \left[1\left(\mathbf{k}_{-} \cdot \mathbf{r}_{\perp}-\omega_{-} t\right)\right]\right\}$

where $\mathbf{r}_{\perp} \equiv x \vec{i}+z \vec{k}$ and $\mathbf{k}_{ \pm} \equiv k_{ \pm} \vec{i} \pm \beta \vec{k}$. We substitute the above ansatz into the Navier-Stokes and continuity equations, retain linear terms in the secondary, and eliminate the disturbance pressure and streamwise component of the velocity. On averaging over $x, z$ and $t$, only the resonant modes survive, which are related as follows:

$$
k_{+}+k_{-}=k, \quad \text { and } \quad\left(\omega_{+}+\omega_{-}\right)_{r}=\omega .
$$

We then obtain

$$
\begin{gathered}
{\left[1\left(\omega_{+}-k_{+} U\right)+\frac{1}{R}\left(D^{2}-k_{+}^{2}-\beta^{2}\right)\right]\left[\left(k_{+}^{2}+\beta^{2}\right) f_{s+}-D v_{s+}\right]-1 k_{+} U^{\prime} v_{s+}-} \\
A_{p}\left(\frac{k_{+}}{2 k_{-}}\right)\left\{\left[1 k_{+} u D+v D^{2}+1 k_{-} D u\right] v_{s-}^{*}+\left[\left(\beta^{2}-k_{-} k_{+}\right) v D+\right.\right. \\
\left.\left.1 k_{+}\left(k_{-}^{2}+\beta^{2}\right) u\right] f_{s-}^{*}\right\}=0
\end{gathered}
$$

and $\left[1\left(\omega_{+}-k_{+} U\right)+\frac{1}{R}\left(D^{2}-k_{+}^{2}-\beta^{2}\right)\right]\left(D f_{s+}-v_{s+}\right)-1 k_{+} U^{\prime} f_{s+}$ 


$$
\begin{gathered}
+A\left(\frac{k+k_{-}}{2}\right)\left[\frac{v}{k_{-}} D v_{s-}^{*}-1 u\left(v_{s-}^{*}+D f_{s-}^{*}\right)\right] \\
+\frac{A}{2}\left[v\left(\frac{k \beta^{2}}{k_{-}}+D^{2}\right)-1 k_{-}(D u)\right] f_{s-}^{*}=0 .
\end{gathered}
$$

The quantity $f_{s+}\left(\equiv-1 w_{s+} / \beta\right)$ is proportional to the spanwise component of the secondary disturbance velocity, and the operator $D$ stands for differentiation with respect to $y$. The boundary conditions are

$$
\mathbf{u}_{s}=0 \quad \text { at } \quad y=0 \quad \& \quad \mathbf{u}_{s} \rightarrow 0 \quad \text { as } \quad y \rightarrow \infty
$$

Equations (5) and (6), along with two corresponding equations in $v_{s-}^{*}$ and $f_{-}^{*}$, describe an eigenvalue problem for the secondary instability. Note that secondary instabilities account for one contribution to nonlinearity, that of the triad interaction between the primary Tollmien-Schlichting mode and two three-dimensional disturbances, the sum of whose wave-numbers equals that of the primary.

A Chebyshev spectral collocation method is used for the numerical solution [13]. A grid stretching given by

$$
y_{j}=\frac{b\left(1+Y_{j}\right)}{1+2 b / y_{\infty}-Y_{j}}
$$

is applied, where

$$
Y_{j}=\cos \left(\frac{\pi j}{N_{g}}\right), \quad j=0,1,2, \ldots, N_{g}
$$

are Chebyshev collocation points. We thus transform the computational domain from $(-1,1)$ to $\left(0, y_{\infty}\right)$, where $y_{\infty}$ is chosen to be at least 5 times the boundary layer thickness, and cluster grid points close to the wall by tuning the parameter $b$. Results for the boundary layer over a flat-plate compare very well with those of [11]. On changing the number of grid points $N_{g}$ from 80 to 160 , the eigenvalues remained identical up to the sixth decimal place.

We are interested here in the flow past flat solid surfaces inclined at an angle $2 m /(m+1)$ to the flow, where the external flow velocity varies downstream as $U_{\infty} \sim x^{m}$ [14]. We consider constant pressure $(m=0)$ and decelerating boundary layers $(m<0)$ in this paper, since the behaviour in the transition zone differs in the two. The profile of mean streamwise velocity for a given $m$ 
is described by the Falkner-Skan equation [15]

$$
f^{\prime \prime \prime}+f f^{\prime \prime}+\frac{2 m}{m+1}\left(1-f^{\prime 2}\right)=0
$$

where $U=f^{\prime}$. The boundary conditions are $f=f^{\prime}=0$ at the wall, and $f^{\prime} \rightarrow 1$ as $y \rightarrow \infty$. Computations of instability have been carried out for a variety of Reynolds number and primary disturbance amplitude. The subharmonic mode, of wavenumber $\left(=k_{+}=k_{-}\right)=k / 2$ is found always to be the most unstable.

\section{The transition zone}

The transition zone is easiest to describe quantitatively in terms of the variation with the streamwise coordinate, $x$ of the intermittency, $\gamma$. Given that the Reynolds number $R(x)$ is the only parameter in the instability problem, it is reasonable to assume that most turbulent spots will originate within a narrow spanwise strip around a particular streamwise location $x_{t}$, denoted here as the location of transition onset. This is the hypothesis of concentrated breakdown [5]. With spots appearing randomly in accordance with a Poisson

distribution in time and a uniform distribution in the spanwise coordinate $z$, $\gamma$ would vary downstream as [5]

$$
\gamma=1-\exp \left[\frac{-n \sigma}{U_{\infty}}\left(x-x_{t}\right)^{2}\right]
$$

or

$$
F \equiv \sqrt{-\log (1-\gamma)}=\sqrt{\frac{-n \sigma}{U_{\infty}}}\left(x-x_{t}\right)
$$

such that the intermittency parameter $F$ varies linearly in $x$. Here $U_{\infty}$ is the free stream velocity, $n$ is the number of spots forming per unit length in the spanwise direction, $z$, per unit time, at $x_{t}$. The shape and downstream growth of turbulent spots is experimentally observed to be similar in all pressure gradients: a turbulent spot maintains an arrowhead shape when viewed from above, and remains self-similar as it grows $[16,17]$. It is convenient therefore to define a non-dimensional spot propagation parameter as

$$
\sigma=\left[\frac{1}{U_{r}}-\frac{1}{U_{h}}\right] U_{\infty} \tan \zeta
$$


where $\zeta$ is the angle subtended by the spot at its origin and $U_{h}$ and $U_{r}$ respectively are the speeds with which its head and rear convect.

It is relatively straightforward to measure other average transition zone quantities such as the burst rate and the persistence time distribution of laminar or turbulent flow as functions of the streamwise distance. The burst rate $B$ is here defined as the mean number of switches from laminar to turbulent flow per unit time at a given location, and the persistence time $W$ of laminar flow is the duration of time for which the flow remains continuously laminar at a given location. The transitional intermittency has been measured in many experiments, but there are no data available for the other measures, as far as we know. It is our suggestion that a lot of information can be gained from knowing how these quantities vary in the streamwise direction. For a Poisson process, the burst rate is related to the local intermittency by

$$
B \propto(1-\gamma)(-\ln (1-\gamma))^{1 / 2}
$$

and the probability density function of persistence time $W$ of laminar flow is

$$
p(W) \propto(1-\gamma)^{W-1}(-\ln (1-\gamma)) .
$$

In quiet facilities, the parameter $F$ in equation 12 in constant pressure flow (to be discussed later) varies almost perfectly linearly with $x$, but its variation in decelerating flows is nonlinear with an increasing slope, as seen in figure 2 The explanation [1] for the nonlinear behaviour of intermittency is that spot birth is not random, but mainly regular, in a pattern dictated by the most unstable secondary mode.

\section{Stochastic Simulations}

Stochastic simulations of the transition zone (using a cellular automaton-like approach) are performed, employing observed properties of spot growth and convection $[1,19]$. We consider two scenarios for spot birth. The first is a random breakdown, as discussed above, with no relation to instability. The second is predominantly regular breakdown, where the pattern prescribed is that of the maxima in disturbance vorticity in the secondary instability. Simulated transition zones for random and regular (harmonic) breakdown are shown schematically figures 3 and 4 respectively.

The zone, as viewed from above, is discretized into $200 \times 400$ rectangular grids in $x$ and $z$. Each grid is assigned an integer $\chi$, equal to 0 if the flow there is laminar and 1 if it is turbulent. A regular breakdown scenario is prescribed as follows. During the first time interval, one spot is generated 


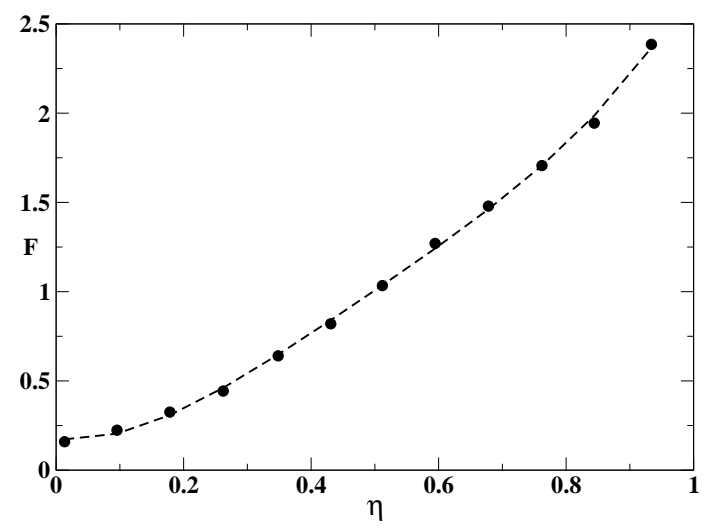

Figure 2. Variation of the intermittency parameter $F$ in adverse pressure gradient, $m=-0.05$. Symbols: experiment [18].

every $N_{z}$ sites, at $X=1$ and $Z=l N_{z}+i, l=0,1,2 \cdots L_{z} / N_{z}$, where $i$ is an integer between 1 and $N_{z}$. The uppercase stands for discretised coordinates. After $N_{t}$ time steps the spots form at spanwise locations which are staggered in the spanwise coordinate with respect to the spots generated earlier, i.e., at $X=1$ and $Z=l N_{z}+j$ for harmonic and $Z=(l+1 / 2) N_{z}+j$ for subharmonic breakdown where $j=i+1$. This prescription corresponds to spots forming at the crests of an oblique wave of spanwise and streamwise wavenumbers

$$
\beta=\frac{2 \pi}{N_{z} \Delta Z} \quad \text { and } \quad k=\frac{2 \pi}{N_{t} \Delta T v}
$$

respectively, where $v$ is the streamwise velocity of the wave crest. The phase of the oblique wave is randomised with a small probability (1\%), and a small fraction $(5 \%)$ of the spots are generated randomly. It is assumed that this prescription mimics qualitatively the randomisation due to external disturbance. Changes in these fractions do not change the answers significantly.

In a random breakdown, spots appear at $X=1$ in accordance with a Poisson distribution in time and a uniform distribution in the spanwise direction. In each case, the downstream growth of turbulent spots follows the same algorithm. During one time interval, the front of each spot moves ahead by 2 grid locations, while the rear moves forward by 1 grid location. The lateral dimension of the spot is increased by 1 grid location on either side. The simulated spot is thus triangular, and retains its shape for all time. A real spot is a 


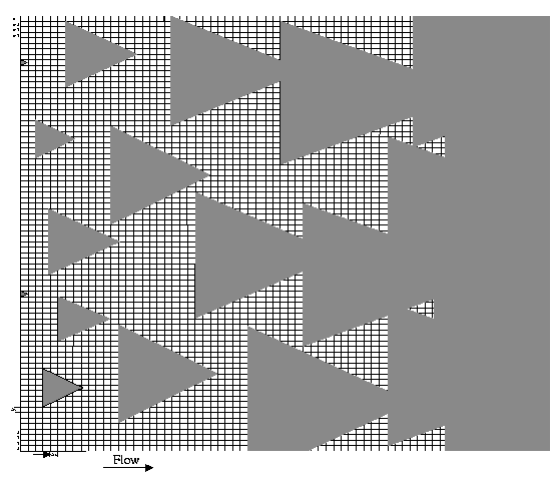

Figure 3. Schematic diagram of the simulation domain in a random spot breakdown scenario. Spots appear according to a Poisson distribution in time, and are uniformly distributed in the spanwise direction.

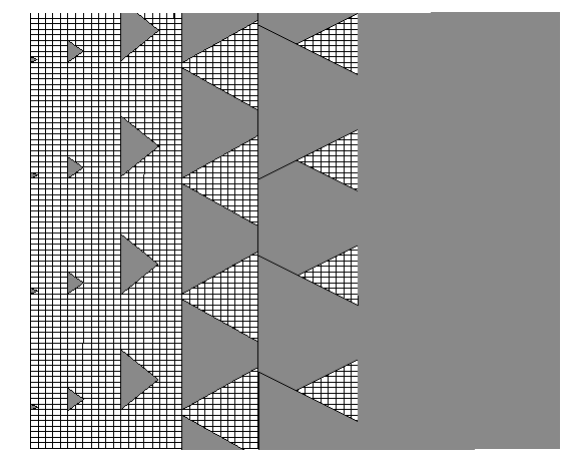

Figure 4. Schematic diagram of the simulation domain in regular breakdown. Spots are formed at regular intervals in the spanwise direction and are staggered after every time period, in a manner dictated by the secondary instability.

blunter at its leading edge than a triangle, but this is assumed not to change the results significantly.

In experiments, the speed of the front of the spot is comparable to the flow velocity $U_{\infty}$ while the rear moves forward approximately at $0.5 U_{\infty}$. The halfangle $\zeta$ subtended by the spot at its origin is about $10^{\circ}$ in a constant pressure boundary layer, and increases with adverse pressure gradient.

In Figs. [5 and 6 it is demonstrated that intermittency behaviour is sensitive to the ratio of spanwise to streamwise wavenumbers. This observation is used to strengthen the connection we make between instability and its signature in the transition zone. The simulations shown in Fig. 5 display a distinct change in slope at $X=13$, just downstream of the location $(X=11)$ at which the heads of a set of spots formed at a time $T$ just touch the rears of the spots 


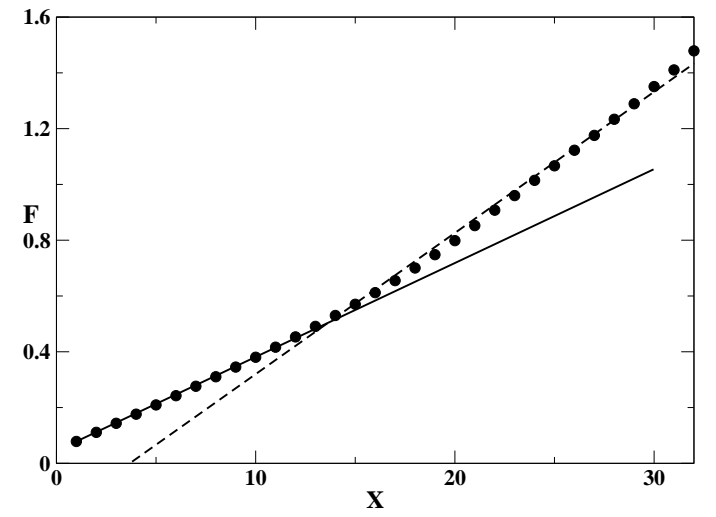

Figure 5. Streamwise variation of the intermittency parameter for $N_{z}=49$ and $N_{t}=10$. Symbols: regular breakdown, solid line: random breakdown. The mean spot generation rate is kept constant at $N=0.01$. The dashed line shows the best linear fit for the downstream part of $F$.

formed at $T-N_{t} \Delta T$. In the simulations of Fig. 6. all parameters have been kept the same, except that the values of $N_{z}$ and $N_{t}$ have been switched. In this case the spots touch each other laterally first (at $X=6$ ) downstream of which a sharp change in slope is again evident. At low $F$, i.e., upstream of spot merger, the intermittency behaviour in each case is exactly the same as that when spots form randomly (with the same breakdown rate). This is not surprising, given that the spots are too small to "see" each other. At higher intermittencies, quite surprisingly, for the same mean breakdown rate and identical spot growth, the length of the transition zone is seen to be very different, which tells us that spot-merger plays a large role in transition. A purely lateral merger results in a much higher degree of overlap (on both sides), and consequently, less of the region is occupied by spots. This of course means that transition to turbulence will proceed much more slowly as shown in Fig. 6. A purely longitudinal merger on the other hand, results in far less spot overlap, meaning more occupancy by spots, and a shortened transition zone (Fig. 5). Incidentally, it is often the case that experimental results seem to lie on two straight lines, as in this figure. This has been termed a 'sub-transition' [20]. Our simulations show that this change in slope in not a result of spots suddenly changing their downstream growth behaviour, but is a natural consequence of their spatial arrangement.

We now ask which kind of merger scenario would be consistent with growing instability modes. To do this, we perform a secondary instability analysis to obtain the streamwise and spanwise wavenumbers for which the secondary wave grows fastest. For example, for an adverse pressure gradient flow of FalknerSkan parameter $m=-0.06$, the maximum growth rate $\left(\omega_{i}\right)$ of the secondary instability wave is 0.067 , and occurs when the wavenumbers $(k$ and $\beta$ ) are 


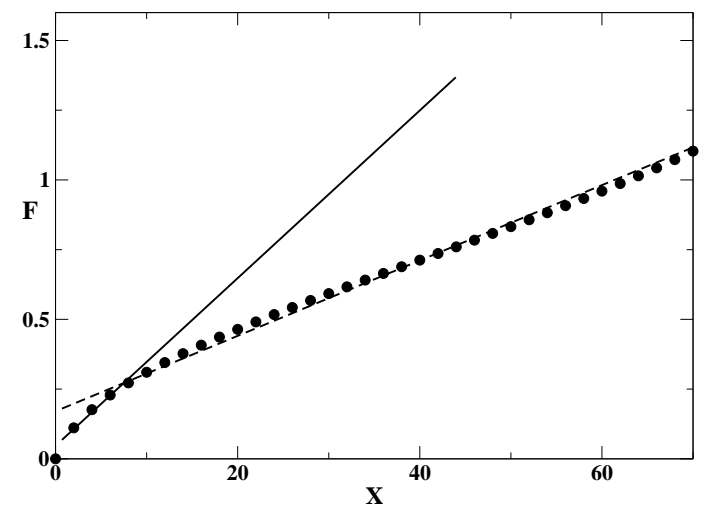

Figure 6. Streamwise variation of the intermittency parameter for $N_{z}=10$ and $N_{t}=49$. Other parameters and notation are the same as in figure 5 It can be seen that the transition zone is much longer in this case.

0.165 and 0.215 respectively. The corresponding phase speed is $0.524 U_{\infty}$. Using the fact that the rear of a spot travels at approximately half the freestream velocity, we have $\tan \zeta=2 \Delta Z / U_{\infty} \Delta T$. The spot propagation angle is taken as $\approx 20^{\circ}$, which is close to the experimental value for this pressure gradient [21], and from equation (16) we may estimate the breakdown ratio $N_{z} / N_{t}$ to be $\sim 4.9$. This ratio changes only by $2 \%$ for a $50 \%$ change in Reynolds number, and may be taken to be independent of Reynolds number.

In figure 7 we consider an adverse pressure gradient, $m=-0.05$. The ratio $N_{z} / N_{t}$ obtained from stability analysis for this case is 3.23 , and in the stochastic simulations we use $N_{z}=39$ and $N_{t}=12$. The analysis is done at a Reynolds number of 220, based on the boundary layer momentum thickness, to match the experimental value. A similar result for $m=-0.06$ is available in [1]. The results of both simulations are in good agreement with experiments. In particular, the changing slope of the intermittency parameter $F(x)$ is followed well. We may conclude that a spot breakdown pattern as prescribed by the secondary instability gives rise to intermittency behaviour in qualitative agreement with measurements in highly decelerating flows.

It may be noticed that there is some discrepancy at the beginning of the transition zone, where it is known that experimental data may be inaccurate: errors could arise unless the data is collected over extremely long times. Secondly, tiny patches of turbulence can be difficult to distinguish from noise. It is also to be remembered that the hypothesis of concentrated breakdown is an idealisation: in reality spots would be forming within a narrow streamwise strip around $x=x_{t}$, rather than at one particular location, this can smear out the intermittency at $\gamma \sim 0$.

The burst rates obtained from the present simulations with a random breakdown are in agreement with equation 14, as seen in figure 8. Any deviation 


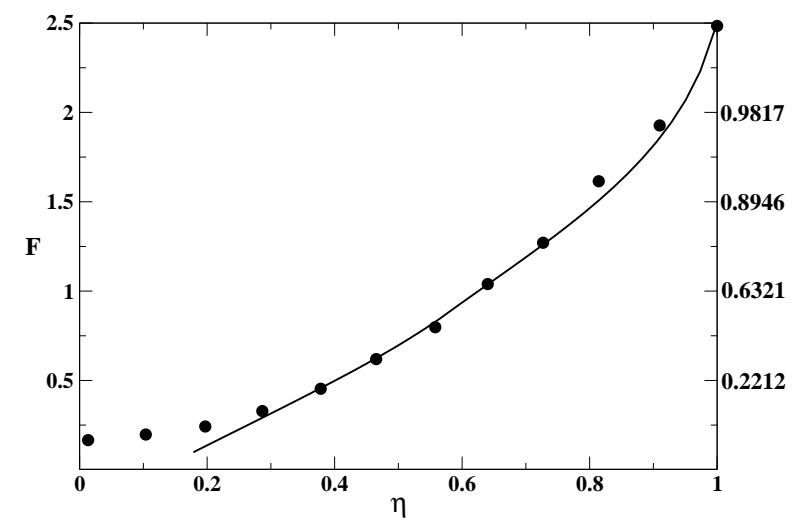

Figure 7. Downstream variation of the intermittency parameter $F$ in an adverse pressure gradient of $m=-0.05$. Solid line: stochastic simulations with $N_{z}=39, N_{t}=12$, from the dominant secondary mode, symbols: experiments [22].

from this behaviour is a sign that spot breakdown is not random. When the breakdown is mostly regular, it is clear that the burst rate distribution is "peaky" and symmetric with respect to $\gamma=0.5$. The burst rate variation with intermittency is similar for sub-harmonic and harmonic type breakdowns. We suggest here that there is a need for experimental measures of these quantities, especially in adverse pressure gradient boundary layers, which will help us quantify the breakdown scenario. The burst rate behaviour displayed when the dominant merger is lateral is qualitatively different from the case where the merger is longitudinal. The burst rates obtained from the simulations whose intermittencies are shown in figure [5 and [6] are plotted in figure 8 , and are a demonstration of this.

The probability density function of the persistence time $W$ is plotted in figure 9. The data is obtained by running the simulation over 20 million time steps (after reaching stationary state), monitoring a particular streamwise location in the middle of the span and collecting statistics of lengths of strings of zeroes between two 1's. The reverse information, about strings of 1's between two zeroes, is less interesting. We present results for $W$ at location where the intermittency is $\gamma=0.1$. For a random spot breakdown, the probability density of the persistence time decays exponentially with persistence time, as is expected from equation (15). At low intermittency levels, in the case of regular breakdown, an overall decay is significantly modulated by ups and downs. At higher levels of intermittency, if the breakdown is predominantly regular, the probability of very large waiting times is extremely low, as expected. The most probable persistence times correspond to the modulated streamwise extent of the laminar zone between two rows of spots. In the case of subharmonic (staggered) spot arrangement, there is a probability of skipping one row of spots, 


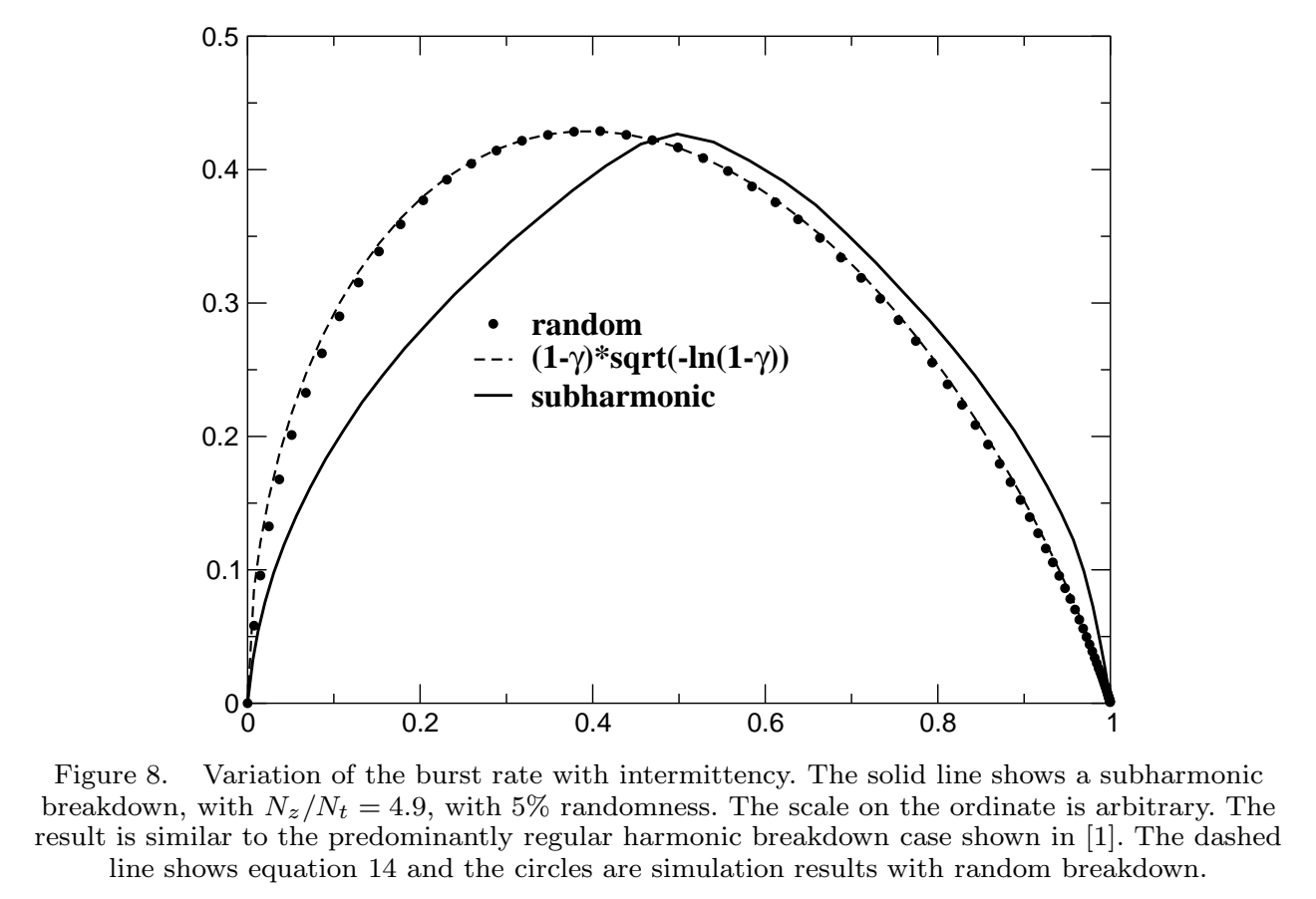

Figure 8. Variation of the burst rate with intermittency. The solid line shows a subharmonic breakdown, with $N_{z} / N_{t}=4.9$, with $5 \%$ randomness. The scale on the ordinate is arbitrary. The result is similar to the predominantly regular harmonic breakdown case shown in [1]. The dashed line shows equation [14 and the circles are simulation results with random breakdown.

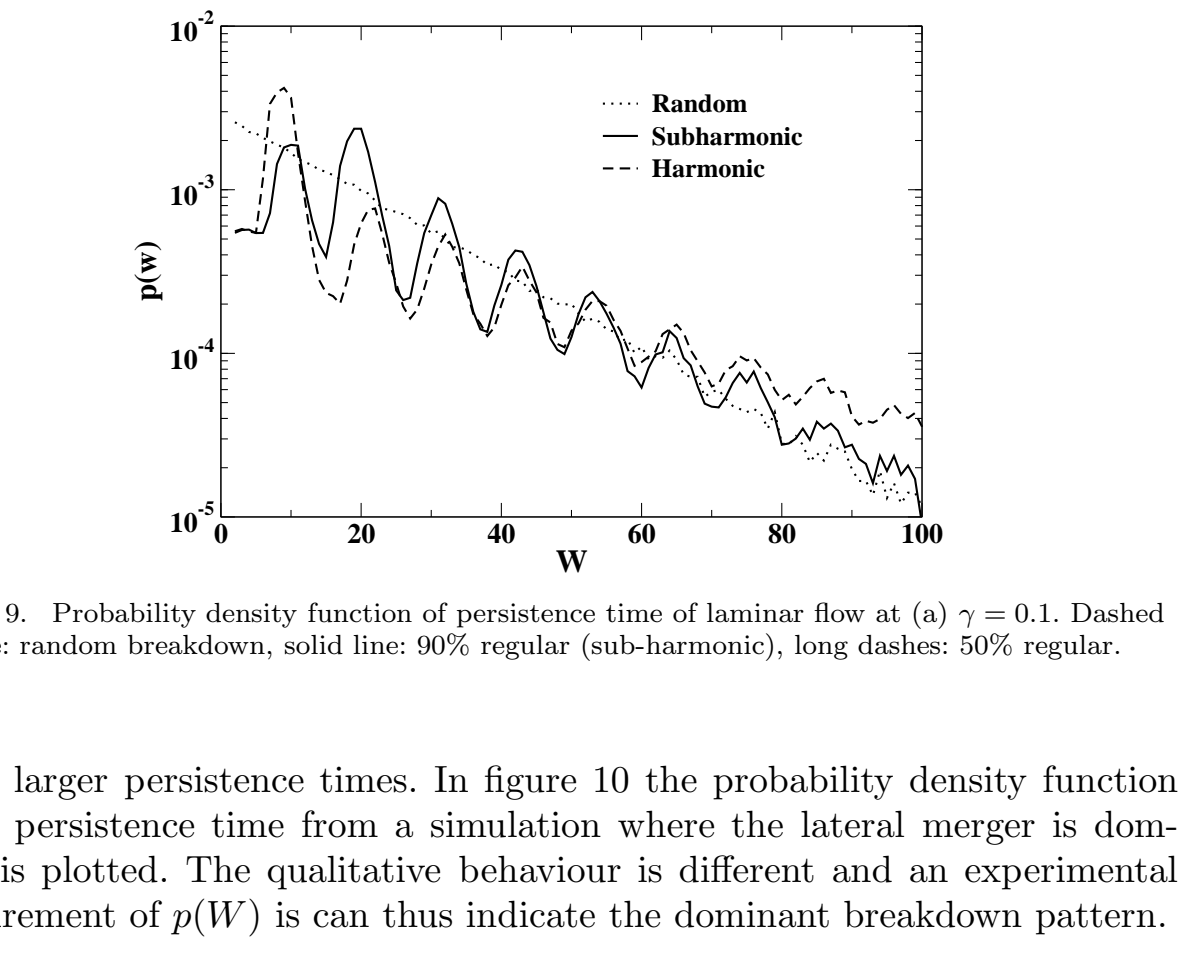

Figure 9. Probability density function of persistence time of laminar flow at (a) $\gamma=0.1$. Dashed line: random breakdown, solid line: $90 \%$ regular (sub-harmonic), long dashes: $50 \%$ regular.

giving larger persistence times. In figure 10 the probability density function of the persistence time from a simulation where the lateral merger is dominant is plotted. The qualitative behaviour is different and an experimental measurement of $p(W)$ is can thus indicate the dominant breakdown pattern.

meastent of $p(W)$ is can the indicate the dominant breakdown pattern.

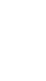

\author{
(1)
}




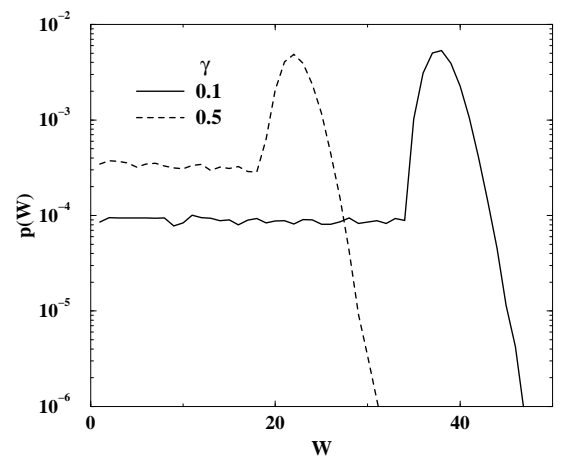

Figure 10. Probability density function of persistence time of laminar flow at $\gamma=0.1$ (solid line) and $\gamma=0.5$ (dashed line), The simulations are carried out under conditions where lateral merger is dominant as in Fig. [6]

\section{$5 \quad$ Is spot birth random in constant pressure gradient flows?}

In constant pressure (flat plate boundary layer) flow, two kinds of behaviour are observed. In high-disturbance environments, the intermittency behaviour is similar to that seen in adverse pressure gradients, except the dominant mechanism is the secondary instability of streaks. The experimentally measured intermittency in such a flow [23] has been shown [1] to be consistent with the dominant instability pattern. Most experiments, however, are conducted in flat plate in environments which are painstakingly maintained quiet. Here, the intermittency parameter $F$ is linear in $x$, consistent with random spot-birth. Do secondary instabilities not directly trigger spot birth here? A partial answer may be gleaned from figure 11] where the spanwise/ streamwise ratio of wavelengths of the dominant mode is shown as a function of the pressure gradient. The ratio decreases with decreasing pressure gradient, and $N_{z} / N_{t}$ is about 2.3 for a constant pressure boundary layer (since now $k=0.085, \beta=0.146$ and $v=0.353$ for the most dangerous mode). For a regular breakdown with this ratio, simulations show that the variation in $F$ is linear up to about $\gamma=0.75$ (figure 12), the slope increases towards the end of the transition zone. On the lines of the discussion on Fig. 5 and 6 we may expect that spot mergers are now a combination of lateral and longitudinal, with longitudinal being marginally dominant. The weakened dominance means that the slope change occurs much closer to full turbulence, at $\gamma \sim 0.8$.

The obliqueness of the most unstable wave provides only a partial answer for why the variation of $F$ is linear in a constant pressure boundary layer. The perfectly linear fit in a vast number of experimental data is also likely to be because the degree of randomness in this case is actually higher than in an adverse pressure gradient boundary layer, as suggested by Gostelow and 


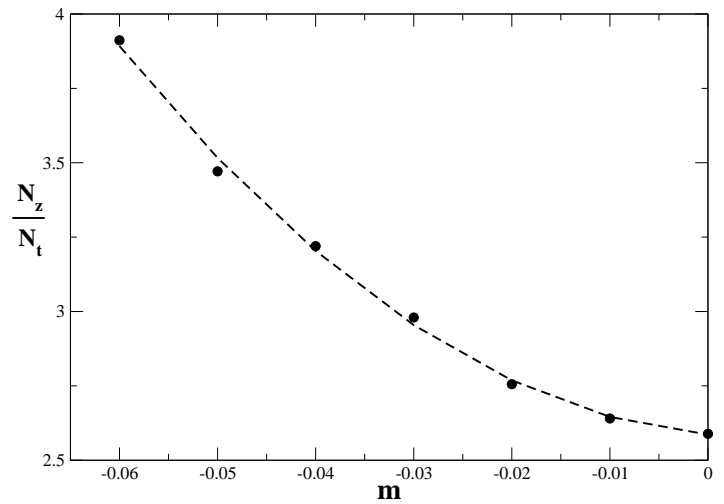

Figure 11. The variation with pressure gradient of the ratio $N_{z} / N_{t}$ as determined by the most unstable secondary mode.

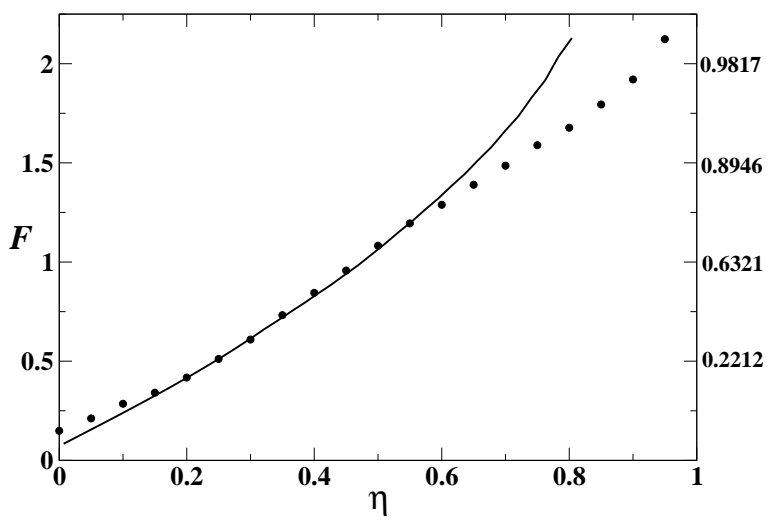

Figure 12. Variation of intermittency parameter $F$ in the boundary layer over a flat plate. Solid line: stochastic simulations with $N_{z}=32, N_{t}=13$ as dictated by secondary instability, the dots are experimental data in constant pressure boundary layers [24].

Walker [25]. The following qualitative arguments indicate why we may expect this.

A decelerating flow is inherently much more unstable [26, 27], as demonstrated in figures 13 and 14. Since the primary wave is slowly growing, we assume that the secondary mode at a given time can be computed by taking the primary wave to be of constant amplitude (this is a temporal analog of the "parallel-flow" assumption often employed in spatially-developing flow). It is to be noted that the growth of the secondary mode can be faster than exponential if the primary mode is unstable as well. Within a very short downstream distance, the disturbance waves in an adverse pressure gradient flow 


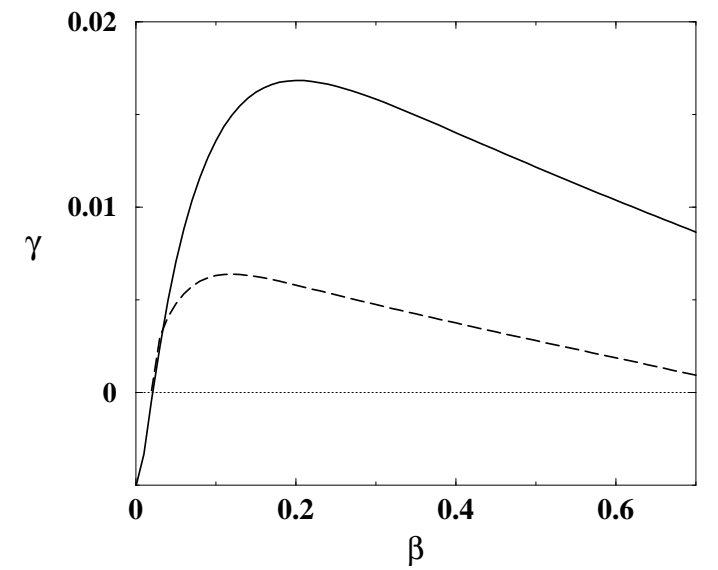

Figure 13 . Growth rate of most dangerous secondary sub-harmonic mode at a Reynolds number

Figure 13. Growth rate of most dangerous secondary sub-harmonic mode at a Reynolds number

$m=-0.06, k_{+}=0.14$, dashed line: $m=0, k_{+}=0.085$.

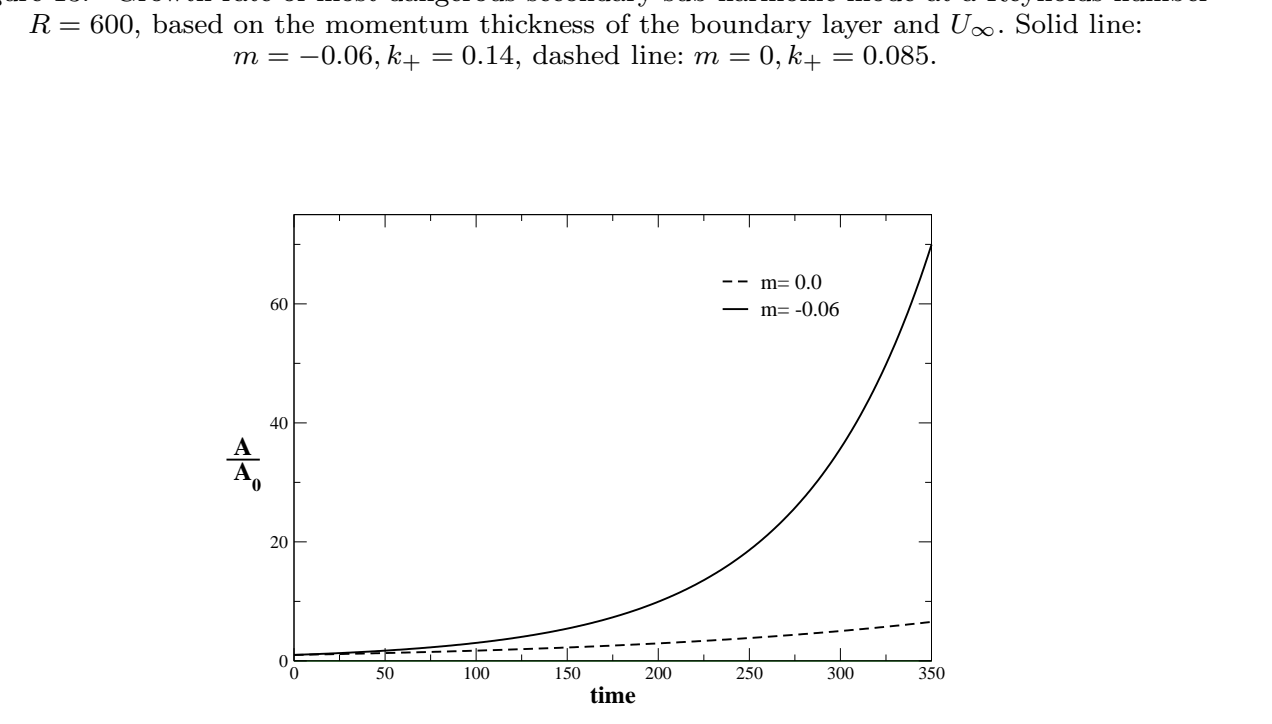

Figure 14. The variation of amplitude of secondary disturbance wave with time in adverse ( $m=-0.06, k=0.185$ and $\beta=0.12$,solid line $)$ and zero $(k=0.13$ and $\beta=0.805$, dashed line $)$ pressure gradient boundary layers.

achieve the threshold amplitude required for breaking down into spots. In the
constant pressure case the attainment of the required threshold is much slower,

achieve the threshold amplitude required for breaking down into spots. In the
constant pressure case the attainment of the required threshold is much slower, offering greater opportunity for stochastic effects.

A significant amplitude modulation of wave packets could be another cause

A significant amplitude modulation of wave packets could be another cause

(16) (1)

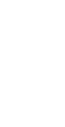

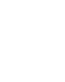

\author{
西

,

,

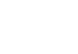

(1)




\begin{tabular}{|c|c|c|c|}
\hline Pressure gradient $(m)$ & Reynolds number & phase speed & group velocity \\
\hline 0 & 200 & 0.412 & 0.295 \\
-0.05 & 200 & 0.492 & 0.655 \\
0 & 600 & 0.353 & 0.188 \\
-0.05 & 600 & 0.510 & 0.666 \\
\hline
\end{tabular}

\begin{tabular}{|c|c|c|c|}
\hline \multicolumn{2}{|l|}{} & \multicolumn{2}{c|}{$k_{+} / \beta$} \\
\hline$A$ & $R$ & $m=0.00$ & $m=-0.049$ \\
\hline 0.01 & 200 & 0.77 & 0.75 \\
0.005 & 200 & 0.91 & 0.70 \\
\hline 0.01 & 600 & 0.65 & 0.70 \\
0.005 & 600 & 0.81 & 0.75 \\
\hline \hline
\end{tabular}

Table 2. Effect of the primary disturbance amplitude on the obliqueness of the most dangerous mode.

for randomness in zero pressure gradient flow. Amplitude modulation would result in different waves reaching the threshold at different streamwise stations, and spot birth would be smeared out over a streamwise distance. In the case of decelerating flow, the group velocities of the waves are much larger, as seen in Table I, and the amplitude modulation takes place over a much narrower width. At a Reynolds number of 200, the group velocity $c_{g}$ of the most unstable secondary in adverse pressure gradient is more than twice the corresponding wave over a flat plate. At a Reynolds number of 600 , this ratio is more than 3. The phase speeds on the other hand are not that different.

Finally, the wavelength ratio of the dominant mode varies significantly with primary disturbance amplitude in flat-plate flow (see [28]). This, however, is not the case in strongly decelerating flow, as seen in Table II. If the external noise is irregular in amplitude, a greater randomisation would take place in flat plate flow than in decelerating flow.

These results indicate that in a decelerating boundary layer, formed by the flow past an inclined plate, the connection between instability and transition is likely to be much easier to observe, and we therefore recommend experimental work in adverse pressure gradient, rather than on flat plate boundary layers.

\section{Effect of concentrated breakdown}

Narasimha [5] proposed the hypothesis of concentrated breakdown in which all spots form within a narrow spanwise belt around the location of transition onset, i.e., within $x_{t} \pm \epsilon$, where $\epsilon<<x_{t}$. While the intermittency behaviour resulting from the hypothesis and the assumption of a random breakdown matches experiment very well in constant pressure and low disturbance en- 
vironments, its validity has been a matter for debate, see e.g. [29]. Since a particular disturbance amplitude is achieved at a particular Reynolds number, it is plausible at least that upstream of a given $x$ location, no spots will form. A large number of spots are likely to form in the vicinity of $x_{t}$, but given that instability modes which are not dominant could continue to grow in the transition zone, a small number could be born at any location downstream. We conduct simulations to estimate how much the intermittency and other parameters depend on making this hypothesis. We do this by allowing an increasing fraction of the spots to form with equal probability anywhere downstream of $x_{t}$. A random breakdown is prescribed. Our conclusion is that even in the unlikely event of a large fraction of spots being born downstream, the intermittency is dominated by spots forming at $x_{t}$, since at any given $x$, these spots are much larger than those born more recently. The intermittency distributions for the extreme cases tried are shown in figure 15. It is seen that even when $80 \%$ spots form downstream of the onset location, the variation of $F$ is still practically linear. However when all the spots are born downstream of $x_{t}$ (as modelled by Emmons [30]), there is a noticeable departure from linearity. In an instability-driven transition, it is highly likely, as discussed above, that a significant fraction of spots will be born around $x_{t}$. It may be noticed that the intermittency behaviour in the case of Emmons breakdown does not appear very different qualitatively from a regular breakdown, and would thus be difficult to distinguish in an experiment. However, an experiment which measures persistence times can distinguish very simply between them, as is evident from figure 16. In an Emmons breakdown, the persistence time distribution decays exponentially, but with a smaller slope than for the concentrated breakdown, as expected.

\section{$7 \quad$ Axisymmetric Boundary Layers}

Transition to turbulence in the boundary layers forming around axisymmetric bodies is poorly understood in spite of wide application in the motion of submarines, fishes etc. When the transverse curvature is significant, transition can proceed quite differently from a two-dimensional boundary layer [20,31]. When the typical patch of turbulence, consisting either of a single spot or a group of spots which have merged laterally, attains a width of the order of the diameter of the cylinder, it wraps itself around the body. Downstream of the location of wrap, further lateral growth is not possible. The turbulent patch then resembles a sleeve [32] displaying only a one-dimensional growth in the streamwise direction.

We have carried out stochastic simulations of the birth and downstream propagation and growth of turbulent spots in the transition zone of an ax- 


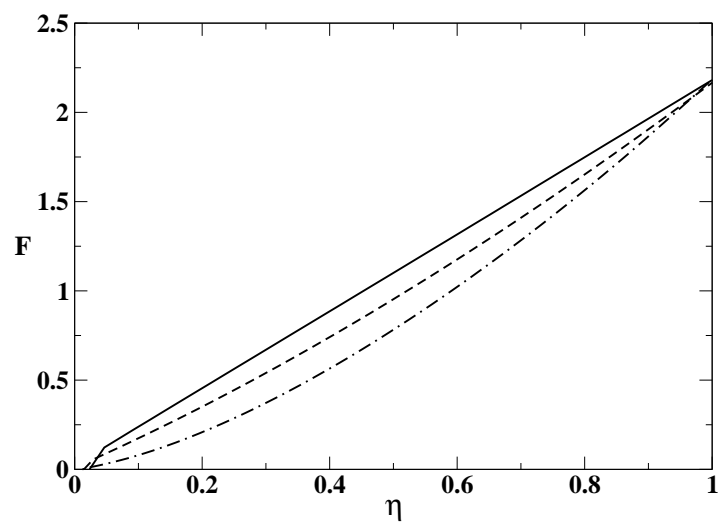

Figure 15. Effect of the hypothesis of concentrated breakdown on the intermittency distribution. Solid line: spots are allowed to form only at $x_{t}$. Dashed line: $80 \%$ spots are born downstream of the onset location. Dot-dashed line: all spots form downstream of the transition onset (Emmons breakdown).

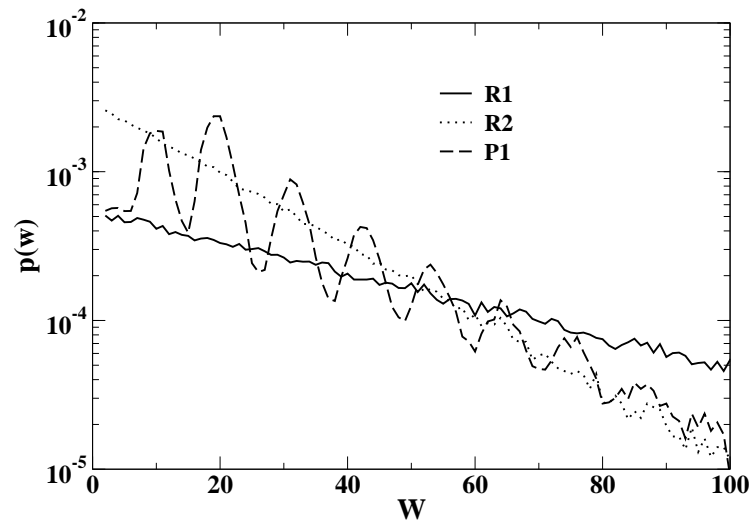

Figure 16. Effect of concentrated breakdown on persistence time distribution. The persistence time is computed at location where intermittency $\gamma=0.1$. The curve marked R2 is according to the hypothesis of concentrated breakdown, R1 is for random breakdown anywhere downstream of $x_{t}$ [30], P1 represents periodic breakdown (sub-harmonic), the pattern is obtained from secondary instability.

isymmetric boundary layer. The downstream variation of the intermittency parameter $F$ is shown in figure 17. The quantity $c$ is the circumference of the body. In the initial region, transition proceeds exactly as it would in twodimensional flow. This is because spots are too small to "see" the body. When spots wrap themselves around the cylinder, a qualitative change is observed, as is expected from the discussion above, and transition proceeds much more slowly after this. The burst rate $B$ shown in figure 18 is another indication of the differences in the transition process. 


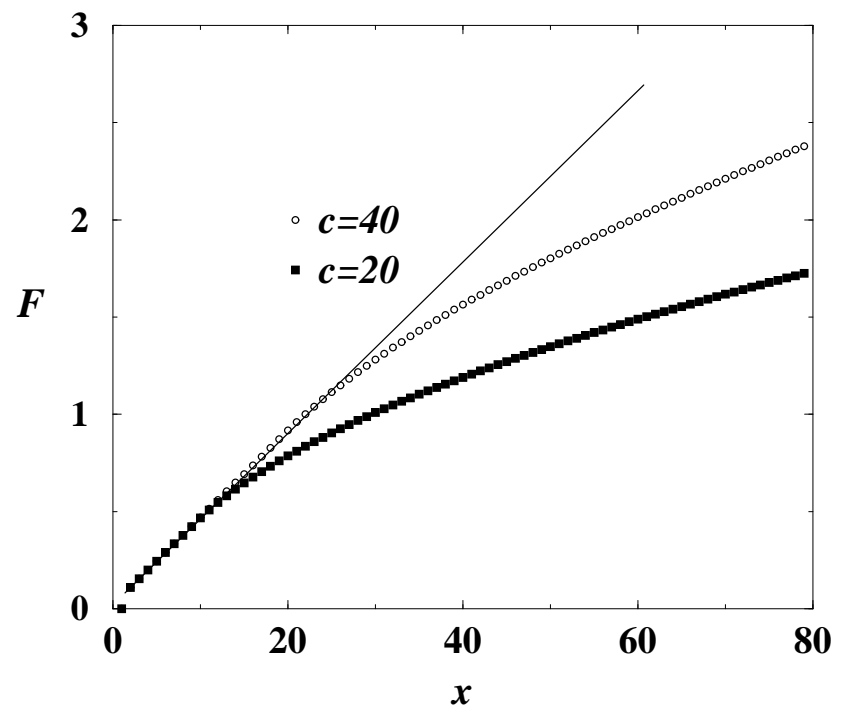

Figure 17. The intermittency factor $F$ Vs. $x$, for different circumferences of the cylinder. The straight line is the result of two-dimensional simulations with the same spot birth rate.

\section{Summary and discussion}

Stochastic simulations, inspired by a cellular-automaton approach, of the generation and propagation of turbulent spots in transitional boundary layers have been conducted, employing the observation that spot growth is self-similar. It is demonstrated that the pattern of spot birth may be inferred from the downstream variation of average quantities in the transition zone, such as the intermittency and the burst rate. This is because the qualitative behaviour in the transition zone depends on whether there is a pattern in spot merger, and if so, whether lateral or longitudinal merger is dominant. Contrary to present belief, our results indicate that relatively simple experiments can tell us a great deal about the connection between instability and transition to turbulence. We show that experiments conducted in decelerating flows are much more conducive to exploring this connection than constant pressure flows. Transverse curvature has the effect of slowing down the transition process.

The simulations of [7] showed a degree of spanwise periodicity in the arrangement of the backward jets (the precursors to spots). Only some of the jets however gave rise to turbulent spots. It has been our contention in TSmode driven transition that the degree of randomness in the birth of spots is expected to decrease as the pressure gradient becomes increasingly adverse. It would be interesting to see whether this is true of bypass transition as well. A direct numerical simulation of an adverse pressure gradient flow should, if this 


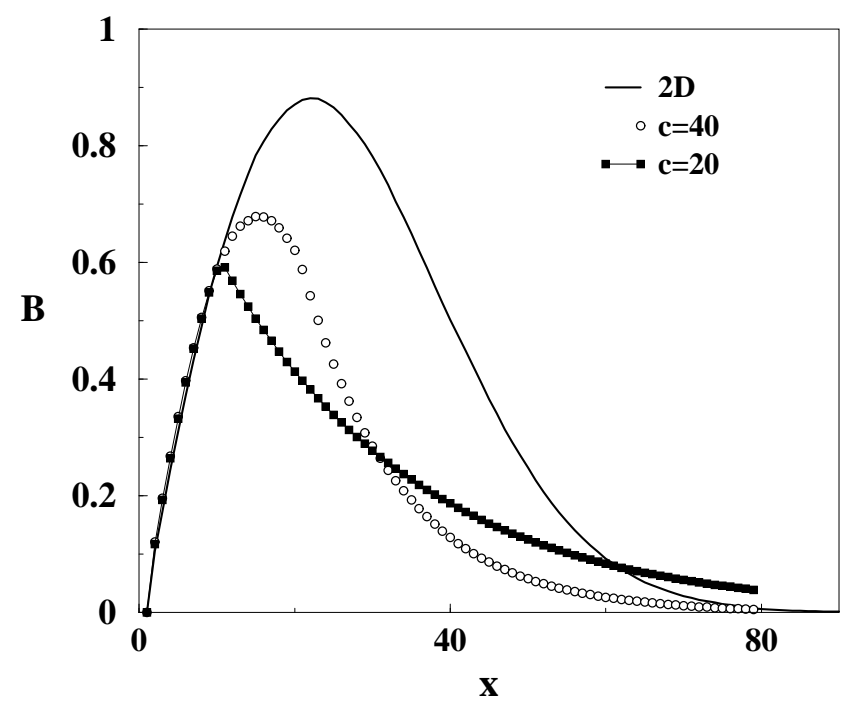

Figure 18. The burst rate in the transition zone of the boundary layer around a cylinder.

contention is right, produce turbulent spots from many more of the reverse jets.

\section{Acknowledgments}

We are most grateful to Prof. Narasimha for suggesting cellular automaton type simulations for the transition zone, and for several discussions. Financial support from the Aeronautical R\&D Board, Government of India, is gratefully acknowledged.

\section{References}

[1] N. Vinod and R. Govindarajan, "Pattern of breakdown of laminar flow into turbulent spots," Phys. Rev. Lett., vol. 93, p. 114501, 2004.

[2] K. H. Bech, D. S. Henningson, and R. A. W. M. Henkes, "Linear and nonlinear development of localised disturbances in zero and adverse pressure gradient boundary layers," Phys. of Fluids, vol. 10, no. 6, pp. 1405-1418, 1998.

[3] H. L. Reed and W. S. Saric, "Linear stability theory applied to boundary layers," Ann. Rev. Fluid Mech., vol. 28, pp. 389-428, 1996.

[4] W. S. Saric, H. L. Reed, and E. B. White, "Stability and transition of three-dimensional boundary layers," Ann. Rev. Fluid Mech., vol. 35, pp. 413-440, 2003.

[5] R. Narasimha, "On the distribution of intermittency in the transition region of a boundary layer," J. Aero. Sci., vol. 24, pp. 711-712, 1957.

[6] A. Prigent, G. Grégoire, H. Chaté, O. Dauchot, and W. van Saarloos, "large sclae finite wavelength modulation within transition shear flows," Phys. Rev. Lett., vol. 89, no. 1, p. 014501, 2002.

[7] R. G. Jacobs and P. A. Durbin, "Simulations of bypass transition," J. Fluid. Mech., vol. 428, pp. 185-212, 2001.

[8] X. WU1, R. G. JACOBS, J. C. R. HUNT, and P. A. DURBIN, "Simulation of boundary layer transition induced by periodically passing wakes," J. Fluid. Mech., vol. 398, pp. 109-153, 1999.

[9] W. M. F. Orr, "The stability or instability of of the steady motions of a perfect liquid and of a 
viscous liquid. Part I: A perfect liquid, Part II: A viscous liquid," Proc. Roy. Irish Acad., vol. 27, no. 3, pp. 69-138, 1907.

[10] A. Sommerfeld, "Ein beitrag zur hydrodynamischen erklaaerung der turbulenten fluessigkeitsbewegungen," Proc. Fourth Internat. Cong. Math., Rome, vol. III, pp. 116-124, 1906.

[11] T. Herbert, "Secondary instability of boundary layers," Annu. Rev. Fluid Mech., vol. 20, pp. 487$526,1988$.

[12] R. Govindarajan, V. S. L'vov, I. Procaccia, and A. Sameen, "Stabilisation of hydrodynamic flows by small viscosity variations," Phys. Rev. E., vol. 67, p. 026310, 2003.

[13] C. Canuto, M. Y. Hussaini, A. Quarteroni, and T. A. Zang, Spectral Methods in Fluid Dynamics. Springer-Verlag, 1st ed., 1987.

[14] G. K. Batchelor, An Introduction to Fluid Dynamics. Cambridge University Press, 2000.

[15] F. M. White, Viscous Fluid Flow. McGraw-Hill, 2 ed., 1991.

[16] R. Narasimha, "The laminar-turbulent transition zone in the boundary layer," Prog. Aero. Sci., vol. 22 , pp. 29-80, 1985.

[17] A. Seifert and I. J. Wygnanski, "On turbulent spots in a laminar boundary layer subjected to a self similar adverse pressure gradient," J. Fluid Mech., vol. 296, pp. 185-209, 1995.

[18] J. P. Gostelow and A. R. Blunden, "Investigations of boundary layer transition in adverse presure gradient," 33rd ASME International Gas Turbine and Aeroengine Congress, Amsterdam (Netherlands), 1988.

[19] N. Vinod and R. Govindarajan, "Instabilities and transition in boundary layers," Pramana, Journal of Physics, vol. 64, no. 3, pp. 323-332, 2005.

[20] R. Narasimha, "Subtransitions in the transition zone," Proc. IUTAM Symp. on LaminarTurbulent Transition, Novosibirsk, 1984

[21] J. P. Gostelow, N. Melwani, and G. J. Walker, "Effect of streamwise pressure gradient on turbulent spot development," ASME paper 95-GT-303, 1995.

[22] J. P. Gostelow, A. R. Blunden, and G. J. Walker, "Effects of free-stream turbulence and adverse pressure gradients on boundary layer transition," Trans. ASME J. Turbomachinery, vol. 116, pp. 392-404, 1994.

[23] M. Matsubara and P. H. Alfredsson, "Disturbance growth in boundary layers subjected to freestream turbulence," J. Fluid. Mech, vol. 430, pp. 149-168, 2001.

[24] S. Dhawan and R. Narasimha, "Some properties of boundary layer flow during transition from laminar to turbulent motion," J. Fluid Mech. , vol. 3, pp. 418-437, 1958.

[25] G. J. Walker and J. P. Gostelow, "Effect of adverse pressure gradients on the nature and length of the boundary layer," Trans. of ASME, vol. 112, pp. 196-205, 1990.

[26] T. C. Corke and S. Gruber, "Resonant growth of three-dimensional modes in Falkner-Skan boundary layers with adverse pressure gradients," J. Fluid Mech. , vol. 320, pp. 211-233, 1989.

[27] C. Liu and S. A. Maslowe, "A numerical investigation of resonant interactions in adversepressure-gradient boundary layers," J. Fluid Mech., vol. 378, pp. 269-289, 1999.

[28] M. B. Zelman and I. I. Maslennikova, "Tollmien-Schlichting-wave resonant mechanism of subharmonic-type transition," J. Fluid. Mech., vol. 252, pp. 449-478, 1993.

[29] M. W. Johnson and A. Fasihfar, "Properties of turbulent bursts in transition boundary layers," Int. J. if Heat and Fluid Flow, vol. 15, no. 4, pp. 283-290, 1994.

[30] H. Emmons, "The laminar-turbulent transition in a boundary layer - part 1," J. Aero. Sci., vol. 18, pp. 490-498, 1951.

[31] R. Govindarajan and R. Narasimha, "Transition delay by surface heating,: a zonal analysis for axisymmetric bodies," J. Fluid Mech., vol. 418, pp. 77-100, 2000

[32] G. N. V. Rao, "Mechanics of transition in an axisymmetric boundary layer on a circular cylinder," Z. Angew. Math. Phys., vol. 25, pp. 63-75, 1974. 